
\title{
Research Suatere \\ All-Optical Tunable Plasmonic Biosensor Made of Graphene and Metamaterial
}

Fariba Lotfi

University of Tabriz Faculty of Physics

Nafiseh Sang-Nourpour

University of Alberta Faculty of Engineering

Reza Kheradmand ( $\nabla$ r_kheradmand@tabrizu.ac.ir)

University of Tabriz https://orcid.org/0000-0001-7117-1921

\section{Research Article}

Keywords: Biosensor, Surface-Plasmon Polariton, Mach-Zehnder interfer-ometer, Graphene, Tunability, Cancer

Posted Date: July 24th, 2021

DOI: https://doi.org/10.21203/rs.3.rs-692445/v1

License: (c) (1) This work is licensed under a Creative Commons Attribution 4.0 International License. Read Full License

Version of Record: A version of this preprint was published at Plasmonics on January 8th, 2022. See the published version at https://doi.org/10.1007/s11468-021-01560-0. 


\title{
All-Optical Tunable Plasmonic Biosensor Made of Graphene and Metamaterial
}

\author{
Fariba Lotfi • Nafiseh Sang-Nourpour • \\ Reza Kheradmand*
}

Received: date / Accepted: date

\begin{abstract}
We demonstrate a novel, label-free and real-time tunable infrared biosensor by employing surface-plasmon polaritons in asymmetric Mach-Zehnder interferometer. The waveguides cladding in the Mach-Zehnder interferometer is made of lossy media with positive and negative electromagnetic susceptibilities, including metamaterial, metal and graphene. The core consists of dielectric media. We introduce two configurations for our biosensor structure. First configuration is an open-path structure and the second one consists of a sample housing made of a silicon layer around the structure. We also present a tunable biosensor by applying a gate voltage to the graphene in the structure. We employ three different cancerous cells, including cervical, breast and basal, as samples to examine the capabilities of the biosensor. Our biosensor structure is highly sensitive, compared to the existing biosensors in the literature, with the sensitivity for basal cancer cell of $1034 \mathrm{THz} / \mathrm{RIU}$. The proposed biosensor structure is compact and easy to fabricate with applications in biomedical sensing and environmental control to detect water pollutants.
\end{abstract}

Keywords Biosensor · Surface-Plasmon Polariton · Mach-Zehnder interferometer · Graphene · Tunability · Cancer

\section{Introduction}

Lable-free plasmonic biosensors are biological systems that have applications in medical analyses [1], drug development [2], food quality control [3] and

F. Lotfi · R. Kheradmand

Optics and Laser Department, Faculty of Physics, University of Tabriz, Tabriz, Iran

E-mail: f.lotfi@tabrizu.ac.ir

E-mail: r_kheradmand@tabrizu.ac.ir (correspondingauthor)

N. Sang-Nourpour

Department of Mechanical Engineering, University of Alberta, Edmonton, Canada

E-mail: sangnour@ualberta.ca 
environmental monitoring [4]. One type of plasmonic biosensors is a refractive index (RI) sensor. The interest in the RI plasmonic sensors is inherent in the sensitivity of surface-plasmon polaritons (SPPs) to the RI of surrounding media [5]. Surface-plasmon polaritons are electromagnetic (EM) excitations that propagate along the conductor-dielectric interfaces. The capabilities of SPP to confine EM energy at sub-wavelength scales make them a suitable candidate for applications in miniaturized plasmonic biosensors [6]. Surfaceplasmon polariton propagation depends on EM susceptibilities of media at the interface. Changing the sign of EM susceptibilities across the interface is the required condition for SPP propagation [7].

Different plasmonic structures are employed in the RI biosensors. One of the simple to design, fabrication and measurement structures is Mach-Zehnder interferometer (MZI) [8]. Mach-Zehnder interferometer provides relatively high sensitivity for the biosensor [9] that makes MZI a suitable sensing platform for biomedical applications [10]. The sensitivity of a MZI-based sensor varies depending on the characteristics of the device such as its media and size [11]. These plasmonic biosensors based on MZI are capable to detect and identify various samples with different RIs, including cancer cells [12].

Cancer is a disease caused by uncontrolled and abnormal cell growth due to genetic and epigenetic defects [13]. The cancerous cells exhibit a high nuclei/cytoplasm rate compared to healthy cells. Therefore, the RI of a cancer cell is higher than normal cells that facilitates employing RI detection approaches to detect cancerous cells [14].

Here, we propose a novel, real-time and label-free plasmonic RI biosensor design made of an asymmetric MZI. The MZI is made of waveguides with lossy dispersive media interfaces with dielectrics and provides a high confinement of EM energy and high propagation length for SPPs. The dispersive media in the biosensor structure is media with different EM susceptibilities, including metamaterial, metal and graphene. For the metal in the biosensor structure we use Ag and for metamterial we employ Fishnet metamaterials [15].

The design of graphene-based plasmonic biosensor proposes a combination of plasmonic response of graphene and metal to improve light-matter interactions. The sole employment of graphene in the plasmonic devices sustains relatively inefficient interaction with light. Therefore, we combine graphene with noble metals, Ag in this paper, to boost the optical response of graphene. To reach a tunable biosensor, we employ graphene and take the advantage of graphene sensitivity to the gate voltage to tune the sensitivity of the biosensor. We employ graphene in the boundary between the core-cladding of metaldielectric waveguides in the biosensor structure.

We introduce two configurations for the biosensor structure to expand biosensor's applicability and functionality. We simulate the results for basal, breast and cervical cancer in our biosensor structure. To examine the specificity of the biosensor structure, we employ cancer samples with RIs near to some normal cells with high growth rates. Our biosensor structure has applications in the identification of different samples including chemical samples with various RIs and biological samples. 
The construction of the paper is as follows. We present the related background to characterize plasmonic biosensors in Sec. 2. We introduce the model of our biosensor structures in Sec. 3. Our simulation results for different configurations are presented and discussed in Sec. 4 . The results are concluded in Sec. 5 .

\section{Background}

In this section, we present the required backgrounds on biosensors and biosensor sensitivity by employing the concept behind SPP propagation along interfaces. We employ linear, homogeneous and isotropic dispersive media interfaces with non-dispersive media in waveguide structures.

Refractive index biosensors made of MZI provide a high sensitivity. The sensitivity of this type of biosensors depends on EM properties of materials in the biosensor structure and geometry of the structure. The RI plasmonic biosensors based on SPP are sensitive to the changes in the RI of surrounding media. Output intensity of MZI changes by employing different media and samples with different RIs in the biosensor structure because of conctructive/destructive interference between SPPs.

The reason for employing different media in the biosensor structure is to analyzed the impact of media with different EM susceptibilities on the biosensor performance and its sensitivity. We employ metal with frequency-dependent permittivity and constant permeability, metamaterial with positive and negative EM susceptibilities [16], and graphene as a dispersive media in the waveguide's cladding.

We employ the generalized Drude-Lorentz model [7] for the EM susceptibilities of metamaterials and metal. The permittivity and permeability in this model convert to each other by applying the duality of electromagnetism [17, 7]. The frequency-dependent permittivity of silicon is described with the Sellmeier equation [18].

Graphene is a monolayer of carbon atoms arranged in a hexagonal lattice with a thickness of $\simeq 0.34 \mathrm{~nm}[19]$. Graphene SPPs have high confinement for EM energy at the interface compared to noble metals. The implementation of graphene facilitates a dynamic tunability for SPP propagation by changing temperature, voltage and chemical potential [20]. Graphene tunability makes this material an attractive candidate to be employed in photonic devices [21].

The EM properties of graphene are characterized by its surface conductivity. The tunability of graphene depends on the surface conductivity which includes intraband $\left(\sigma_{\text {intra }}\right)$ and interband $\left(\sigma_{\text {inter }}\right)$ transitions [22]. Kubo's formula for the graphene surface conductivity is

$$
\begin{gathered}
\sigma_{\text {intra }}(\omega)=\frac{2 i k_{\mathrm{B}} T e^{2}}{\pi \hbar^{2}\left(\omega+{ }^{i} / \tau\right)} \ln \left[2 \cosh \left(\frac{E_{\mathrm{f}}}{2 k_{\mathrm{B}} T}\right)\right] \\
\sigma_{\text {inter }}(\omega)=\frac{e^{2}}{4 \hbar}\left[\frac{1}{2}+\frac{1}{\pi} \arctan \frac{\hbar \omega-2 E_{\mathrm{f}}}{2 k_{\mathrm{B}} T}-\frac{i}{2 \pi} \ln \frac{\left(\hbar \omega+2 E_{\mathrm{f}}\right)^{2}}{\left(\hbar \omega-2 E_{\mathrm{f}}\right)^{2}+\left(2 k_{\mathrm{B}} T\right)^{2}}\right]
\end{gathered}
$$


Here $\omega$ is the angular frequency, $T=300 \mathrm{~K}$ is the room temperature, $k_{\mathrm{B}}$ is the Boltzmann's constant, e is the charge of an electron, $\hbar$ is the Planck's constant, $E_{\mathrm{f}}$ is the chemical potential and $\tau=\mu E_{\mathrm{f}} / v_{\mathrm{f}}^{2}$ is the relaxation time. The electron velocity and mobility in graphene are $v_{\mathrm{f}}=10^{6} \mathrm{~ms}^{-1}$ and $\mu=$ $1 \mathrm{~m}^{2} V^{-1} \mathrm{~s}^{-1}$, respectively [23]. The relation between gate voltage $\left(V_{\mathrm{g}}\right)$ and the chemical potential of graphene is $E_{\mathrm{f}}=\hbar v_{\mathrm{f}} \sqrt{\pi \alpha_{0} V_{\mathrm{g}}}, \alpha_{0}=9 \times 10^{16} \mathrm{~m}^{-2} V^{-1}[22]$.

Sensitivity and specificity are two important factors to specify the capability and functionality of a biosensor at different circumstances. The sensitivity of the biosensor is characterized by $S=\Delta f / \Delta n$ that is the frequency shift per unit RIU. Sensitivity specifies the frequency shift of the transmission spectra $(\Delta f)$ when the RI of sensing sample changes from $n_{1}$ to $n_{2}\left(\Delta n=n_{2}-n_{1}\right.$ ) [24]. We analyze biosensor sensitivity $S$ for different samples RIs. The change in samples RI affects the interference between SPPs that changes the sensitivity of the biosensor.

\section{Model}

We design a plasmonic RI biosensor based on MZI. Owing to SPPs inherent sub-wavelength confinement, SPPs are used in different structures such as biosensors. Our plasmonic biosensor structure consists of an asymmetric MZI made of slab waveguides with lossy dispersive media in the cladding and lossless non-dispersive media in the core of waveguides. We limit our choice of media to dispersive and non-dispersive linear, homogeneous and isotropic materials. The dispersive media in the structure are conductors including metal, metamaterial [25], silicon and graphene [26]. Waveguide's core is made of air with $\varepsilon \simeq 1$ and $\mu \simeq 1$.

To derive metamaterials permittivity and permeability we employ equivalent resistor-inductor-capacitor circuit equation [15]. Metamaterials permittivity and permeability depend on metamaterial's structure parameters [15]. The metamaterial parameters in this paper are $F_{\mathrm{e}}=1, F_{\mathrm{m}}=0.8, \omega_{0_{\mathrm{e}}}=0$, $\omega_{0 \mathrm{~m}}=0.02 \omega_{\mathrm{e}}, \omega_{\mathrm{e}}=1.37 \times 10^{16} \mathrm{~s}^{-1}$ and $\Gamma_{\mathrm{m}}=\Gamma_{\mathrm{e}}=2.73 \times 10^{13} \mathrm{~s}^{-1}[27]$. We also employ noble metal $\mathrm{Ag}$ as a dispersive media in the waveguide structure. The metal parameters are $F_{\mathrm{e}}=1$ and $\omega_{0_{\mathrm{e}}}=0$ [16]. The graphene-based plasmonic biosensor consists of a monolayer of graphene in the metal-dielectric waveguides core-cladding boundary.

Our biosensor structure is made of an asymmetric MZI to generate constructive or destructive interference between SPPs coming from the up and down branches of MZI at the output of the structure. We introduce two configurations for our biosensor structure. First biosensor configuration is an openpath biosensor with the biosensor floated in the sample. The second biosensor configuration is surrounded by a silicon layer, making a sample housing where the sample infiltrates. In the two configurations, different sample RIs and different distances from the sample to the up and down branches of MZI affect the constructive and destructive interference. 
The first configuration of biosensor structure is as Fig. 1. In this biosensor configuration, the biosensor structure is floated in the sample without a sample housing. This open-path biosensor configuration is used for liquid, solid and gas samples.

The second configuration is surrounded by a silicon layer is Fig. 2. In this structure, the sample infiltrates into the sample housing [28]. Due to the presence of a silicon layer around the structure, the sample is restricted to liquid and gas. The presence of a silicon layer around the structure allows having a specific sample housing with a specific size, which gives a control over the biosensors operation. The asymmetric structure of the biosensor and the presence of silicon around the biosensor affect SPP propagation along waveguides. The transmission spectra of the biosensor are affected by these changes in the SPP propagation.

We apply an input laser to the input port of the waveguide as Fig. 1 and Fig. 2. The input laser is a linearly polarized signal with $\boldsymbol{E}=50 \mathrm{~V} / \mathrm{m}$ at frequency range between $0.01 \omega_{\mathrm{e}}$ and $0.06 \omega_{\mathrm{e}}$. From the coupling between the incident EM radiation with the oscillating electrons at the conductordielectric interface, SPPs propagate along the structure. We then analyze the transmission spectra from the output port of MZI.

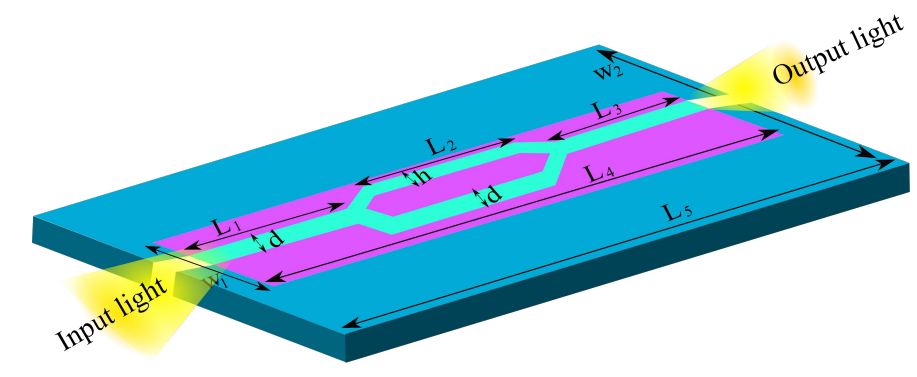

Fig. 1 Schematic of the first configuration of biosensor made of plasmonic waveguides in MZI. The cyan, pink and blue areas are dielectric, dispersive media (including metal, metamaterial and graphene) and sample, respectively. The geometric parameters of the biosensor are $\mathrm{L}_{1}=\mathrm{L}_{3}=350 \mathrm{~nm}, \mathrm{~L}_{2}=400 \mathrm{~nm}, \mathrm{~L}_{4}=1200 \mathrm{~nm}, \mathrm{~L}_{5}=1800 \mathrm{~nm} \mathrm{w}_{1}=320 \mathrm{~nm}$ and $\mathrm{w}_{2}=800 \mathrm{~nm}$. The width of MZI up branch is $\mathrm{h}=35 \mathrm{~nm}$, the width of waveguide core is $\mathrm{d}=50 \mathrm{~nm}$ 


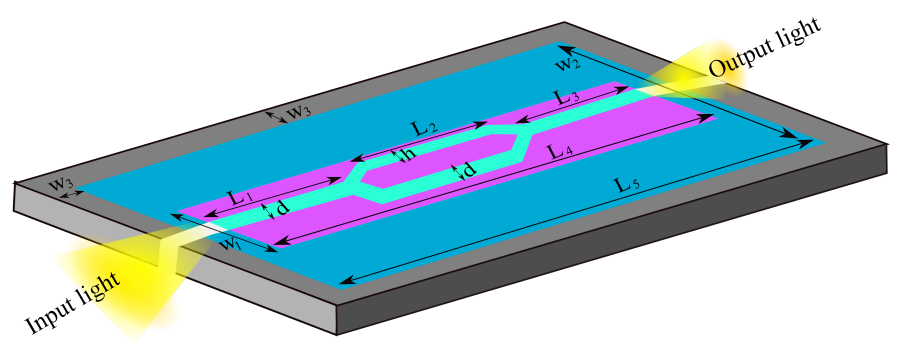

Fig. 2 Schematic of the second configuration of biosensor made of plasmonic waveguides in MZI. The cyan, pink, blue and gray areas are dielectric, dispersive media (including metal, metamaterial and graphene), sample and silicon, respectively. The blue sample housing in second configuration is limited by a silicon layer represented by a gray color. In this configuration, $\mathrm{w}_{3}=75 \mathrm{~nm}$ and other geometric parameters of the biosensor are the same as Fig. 1.

We apply the Finite Element Method to solve TM SPP dispersion equation in the biosensor structures. This method is suitable for different shapes with different sample RIs. We monitor the transmission spectra of the biosensor structure by changing the sample RI. The sensitivity of the biosensor is calculated from the data in the transmission spectra.

We analyze biosensor sensitivity for different RIs of the sample. To show the sensitivity of biosensor, we choose samples with RIs ranging from 1.33 to 1.39 , which are RIs around water RI. However, our biosensor structure is not limited to these RIs. We employ different cancer samples including breast, basal and cervical cancers, as instances, to evaluate the performance of the biosensor. The numerical results related to these descriptions are presented in Sec. 4 .

\section{Results and Discussion}

In this section, we present the results obtained from the simulation of our plasmonic biosensor. We employ metamaterials, metal and graphene interfaces with dielectric in both biosensor configurations. Our plasmonic biosensor is sensitive to the changes in the RIs of the surrounding media. As a result, by varying the sample RI, the transmission spectra of the biosensor shift to different frequencies.

Our biosensor structure is capable to work with various samples with different RIs. As instances, we choose samples with RIs ranging from 1.33 to 1.39. This choice of RI covers a wide range of samples including water and breast, basal and cervical cancers. To characterize the sensing capabilities of the biosensor, we first measure the frequency shift for each RI from the transmission spectra and verify if there is a shift to higher or lower frequencies. We then calculate the sensitivity from the frequency shift for $\Delta n$. The variation 
of frequency shift by $\Delta n$ can have linear or exponential forms. For the linear behavior, we apply a linear fitting to the data to obtain the slope $(\mathrm{S})$.

With these explanations, we are now equipped to give detailed analysis for the two configurations of the biosensor in Subsecs. 4.1 and 4.2. To choose which configurations is more sensitive to the sample RI, we compare the results for the two configurations in Subsec. 4.3. Subsections. 4.4 and 4.5 include analyzing cancer samples and tunability and specificity of the biosensor, respectively.

\subsection{First Configuration: Sensor Floated Inside the Sample}

We present the simulation results for the first configuration of the biosensor in this section. Here, the biosensor is floated inside the sample, as shown in Fig. 1. We employ metal, metamaterial and graphene at the cladding of waveguides in the biosensor structure as follows.

\subsubsection{Biosensor with Metal at the Cladding of Waveguides}

We employ metal at the cladding and air in the core of plasmonic waveguides in the biosensor structure. Metal has negative permittivity and positive permeability. For the Ag metal employed in this paper, the frequency range of negative permittivity is $\omega=0.01 \omega_{\mathrm{e}}$ to $1 \omega_{\mathrm{e}}$ and magnetic permeability is constant $(\mu=1)$. For this metal-dielectric structure, TM SPP propagates along the waveguides from $0.01 \omega_{\mathrm{e}}$ to $0.05 \omega_{\mathrm{e}}[16]$, therefore, we focus on this frequency range.

The magnetic field profile of TM SPPs propagating along the interfaces of biosensor structure at $\omega=0.02 \omega_{\mathrm{e}}$ with sample RI of 1.35 is as Fig. 3(a). In Fig. 3(a), there is a coupling between SPPs propagating along the MZI and the sample that surrounds the structure. This coupling causes a change in the SPP propagation along MZI that changes the interference between SPPs from up and down branches of MZI at the output port. Therefore, the presence of the sample around the entire structure changes the transmission spectra of the biosensor.

We also present the effect of varying sample RI on the transmission spectra of the biosensor. The samples RI varies from 1.33 to 1.39, as explained in Sec. 3. There is red-shift in the transmission spectra of biosensor for different RIs of the sample, as presented in Fig. 3(b).

We calculate the sensitivity $(\mathrm{S})$ of the sensor from the transmission spectra. For the dip in Fig. 3(b), specified by a black arrow, we record the frequency shift by changing the sample RI as shown in Fig. 3(c). In our calculations for the sensitivity, we choose dips or peaks of the transmission spectra that reflects a clear shift in the transmission spectra. The behavior of data is linear in Fig. 3(c). Therefore, we obtain $S$ (slope in Fig. 3(c)) by applying a linear fitting to the data. The sensitivity of the biosensor is $219.75 \mathrm{THz} / \mathrm{RIU}$ when $n$ 
changes from 1.33 to 1.39. This amount of sensitivity is high compared to the previous results in the literature $[5,29]$.
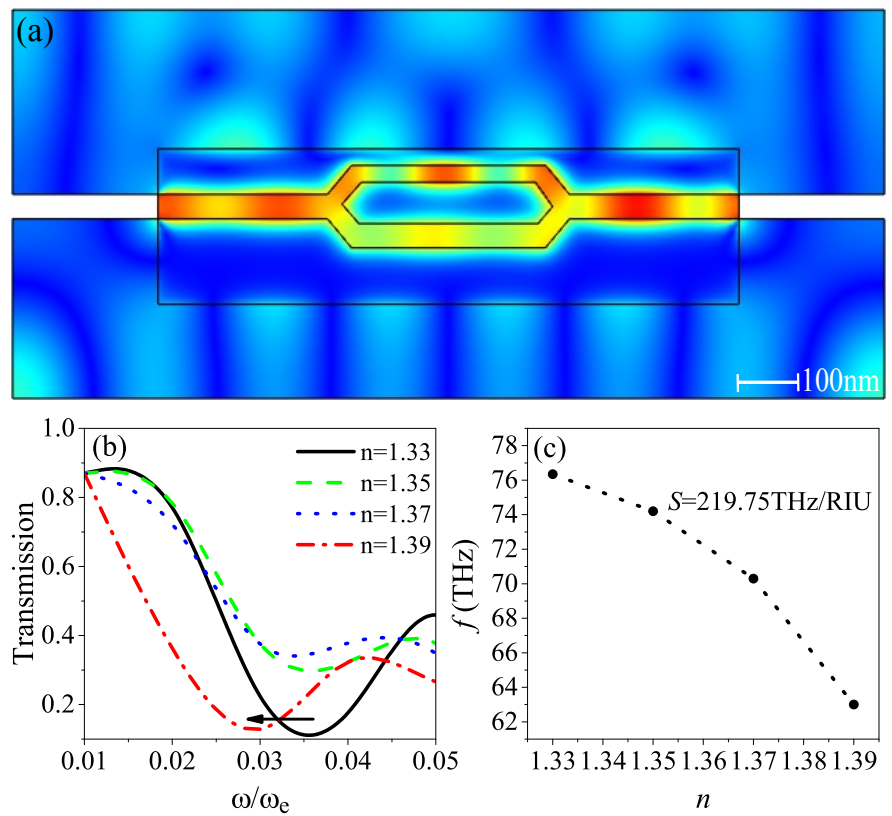

Fig. 3 (a) Magnetic field profile of TM SPPs at $\omega=0.02 \omega_{\mathrm{e}}$ with sample RI of 1.35 , (b) transmission spectra and (c) sensitivity of the biosensor with metal at the cladding of the waveguides, when the biosensor is floated in the sample, by sweeping sample RIs from 1.33 to 1.39 . There is a red-shift in the transmission spectra, as shown with the arrow.

4.1.2 Biosensor with Metamaterial at the Cladding of Waveguides

Here, we employ metamaterial at the cladding of plasmonic waveguides, as an example of media with different EM properties, to check the sensitivity of the biosensor. Metamaterial with negative permittivity and positive permeability supports TM SPPs along the structure [7]. For Fishnet metamaterials with the structural parameters specified in Sec.3, TM SPPs propagate from $0.045 \omega_{\mathrm{e}}$ to $0.054 \omega_{\mathrm{e}}$ [16]. Therefore, we focus on this frequency range to analyze the sensitivity of the biosensor. 


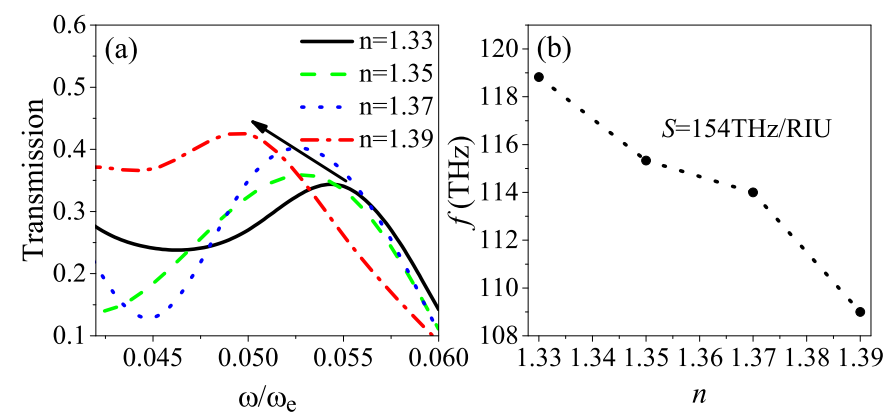

Fig. 4 (a) Transmission spectra and (b) sensitivity of the biosensor with metamaterial at the cladding of the waveguides, when the biosensor is floated in the sample, by sweeping sample RI from 1.33 to 1.39 . There is a red-shift in the transmission spectra, as shown with the arrow.

The transmission spectra of the biosensor has a red-shift by increasing the sample RI from 1.33 to 1.39 , as Fig. 4(a). Figure. 4(b) is the frequency shift in the specified peak (black arrow in Fig. 4(a)) of the transmission spectra by varying RIs from 1.33 to 1.39 and by employing a linear fitting. By applying this linear fitting, the sensitivity of the biosensor is $154 \mathrm{THz} / \mathrm{RIU}$ that is a high sensitivity compared to the previous results in the literature [30,31].

\subsubsection{Biosensor with Graphene in the Boundary Between Core and Cladding} of Waveguides

We employ plasmonic biosensor with graphene in the core-cladding boundary of metal-dielectric waveguides to enhance the biosensor sensitivity arising from the strong energy confinement of graphene. The optical properties and conductivity of graphene are described by the Kubo's formula (Equations (1) and (2)). To have TM SPPs propagating along the interfaces, graphene must behave like a conductor [23]. To achieve this condition, the imaginary part of the surface conductivity must be positive in the operating frequencies.

Here, we present the simulation results for the transmission spectra and sensitivity of the graphene-based biosensor structure. We expect this structure to be more sensitive compared to the metal and metamaterials-based structures, due to the high confinement of energy at the boundary. 

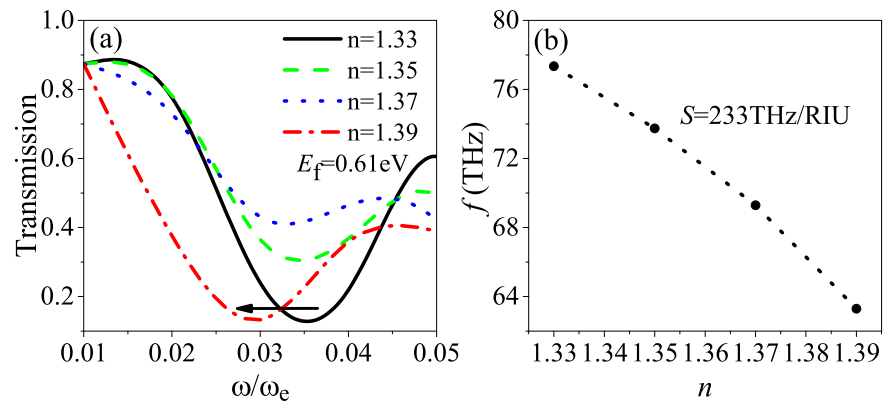

Fig. 5 (a) Transmission spectra and (b) sensitivity of the biosensor with a monolayer of graphene in the boundary between core and cladding of metal-dielectric waveguides, when the biosensor is floated in the sample, by sweeping the sample RI from 1.33 to 1.39 for $E_{\mathrm{f}}=0.61 \mathrm{eV}$. There is a red-shift in the transmission spectra, as shown with the arrow.

The coupling between SPPs propagating along the structure and the sample changes the interferences between SPPs at the output port of MZI. The transmission spectra have a red-shift by increasing the sample RI from 1.33 to 1.39, for $E_{\mathrm{f}}=0.61 \mathrm{eV}$, as Fig. 5(a). By applying a linear fitting to the data for the specific dip in the transmission spectra, the calculated $S$ from the slope of Fig. $5(\mathrm{~b})$ is $233 \mathrm{THz} / \mathrm{RIU}$. This is a high amount of sensitivity compared to the previous results in the literature $[32,33]$.

\subsection{Second Configuration: Sensor with a Sample Housing}

In the second configuration, the biosensor is enclosed with a silicon layer as shown in Fig. 2. To drain the sample into the sample housing, we infiltrate the sample between waveguides cladding and the silicon layer. This configuration is beneficial when sensing liquid/gas samples. The sample is taken in a fluid/gas form and is infiltrated into the sample housing. This approach can be experimentally done by capillary force [34] and selective infiltration methods [35]. We employ different media including metal, metamaterial and graphene at the waveguides cladding and the waveguides core is air. 


\subsubsection{Biosensor with Metal at the Cladding of Waveguides}
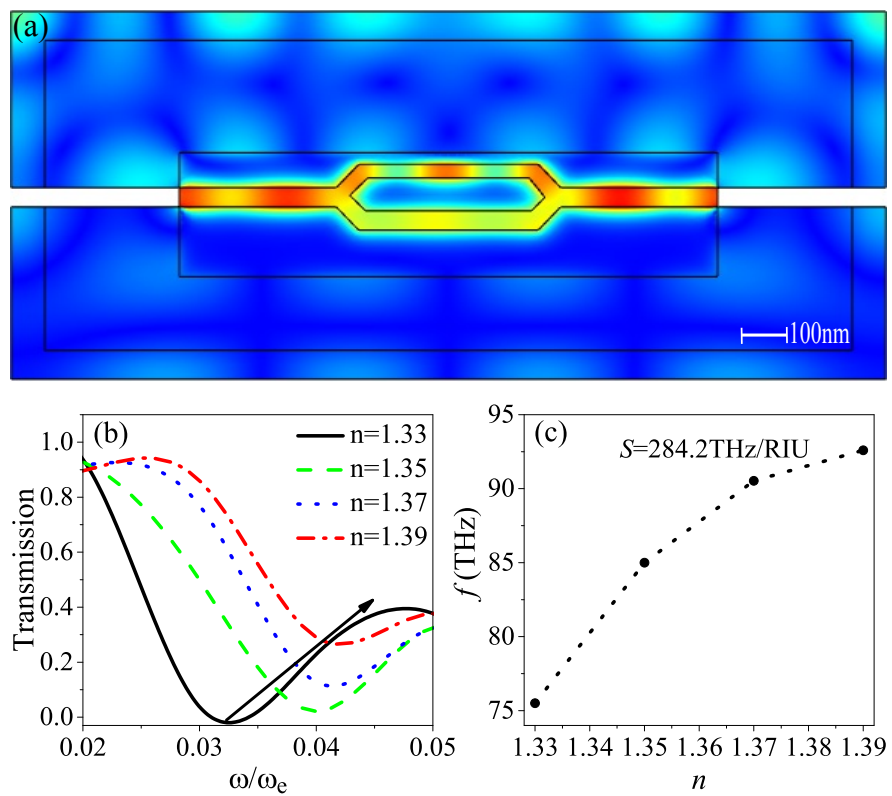

Fig. 6 (a) Magnetic field profile of TM SPPs at $\omega=0.02 \omega_{\mathrm{e}}$ with sample RI of 1.35 , (b) transmission spectra and (c) sensitivity of the biosensor with metal at the cladding of the waveguides, when sample is infiltrated into the sample housing. There is a blue-shift in the transmission spectra by sweeping the sample RI from 1.33 to 1.39 , as shown with the arrow.

Here, we employ biosensor with metal at the cladding of waveguides in the second configuration. The magnetic field profile for TM SPPs along the metaldielectric biosensor structure is as Fig. 6(a) at $\omega=0.02 \omega_{\mathrm{e}}$ with the sample RI of 1.35. In Fig. 6(a), there is a coupling between SPPs propagating along the MZI and the sample around the structure in the sample housing. Therefore, the presence of sample and a silicon layer around the biosensor structure affects the transmission spectra of the biosensor and its sensitivity.

Here, we examine the effect of varying sample RIs on the transmission spectra of the biosensor. The RI of the samples vary from 1.33 to 1.39. Figure. 6(b) shows a blue-shift in the transmission spectra for different RIs of the sample. We choose a specific dip in Fig. 6(b) (as determined by the black arrow) and measure the frequency shift of that dip by changing the sample RI. By applying a linear fitting to the data in Fig. 6(c), the sensitivity is $284.2 \mathrm{THz} / \mathrm{RIU}$, which is a high sensitivity compared to the previous results in the literature [36]. 
4.2.2 Biosensor with Metamaterial at the Cladding of Waveguides

Here, we examine the effect of varying the sample RI on the transmission spectra of the biosensor made of metamaterial cladding. The RI of the samples vary from 1.33 to 1.39 . Figure. 7 (a) shows a blue-shift in the transmission spectra for different RI of the sample. The sensitivity is $144.45 \mathrm{THz} / \mathrm{RIU}$ as shown in Fig. 7(b) that is a high sensitivity compared to the previous results in the literature $[30,31]$.
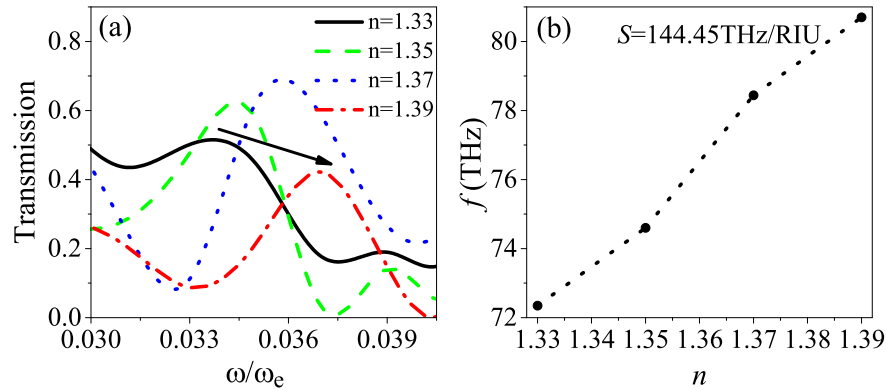

Fig. 7 (a) Transmission spectra and (b) sensitivity of the biosensor with metamaterial at the cladding of the waveguides, when sample is infiltrated into the sample housing. There is a blue-shift in the transmission spectra, as shown with the arrow.

4.2.3 Biosensor with Graphene in the Boundary Between Core and Cladding of Waveguides
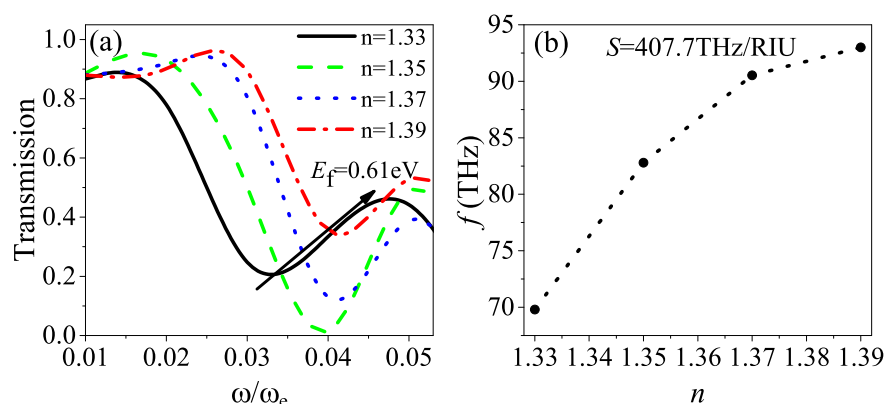

Fig. 8 (a) Transmission spectra and (b) sensitivity of the biosensor with a monolayer of graphene in the boundary between core and cladding of waveguides, when sample is infiltrated into the sample housing, by sweeping the sample RI from 1.33 to 1.39 for $E_{\mathrm{f}}=$ $0.61 \mathrm{eV}$. There is a blue-shift in transmission spectra, as shown with the arrow.

We employ graphene in the boundary between core and cladding of metaldielectric waveguides in the biosensor structure to attain a high energy con- 
Table 1 Comparison between the sensitivity of the first and second configurations

\begin{tabular}{llll}
\hline & $\begin{array}{l}\text { Metal } \\
(\mathrm{THz} / \mathrm{RIU})\end{array}$ & $\begin{array}{l}\text { Metamaterial } \\
(\mathrm{THz} / \mathrm{RIU})\end{array}$ & $\begin{array}{l}\text { Graphene } \\
(\mathrm{THz} / \mathrm{RIU})\end{array}$ \\
\hline $\begin{array}{llll}\text { First Configuration } \\
\text { Second Configuration }\end{array}$ & $\begin{array}{l}219.75 \\
284.2\end{array}$ & 154 & 233 \\
\hline
\end{tabular}

Table 2 RI for basal, breast and cervical cancer and normall cells [28]

\begin{tabular}{lll}
\hline Cell Name & RI of Normal Cell & RI of Cancer Cell \\
\hline Basal & 1.36 & 1.38 \\
Breast & 1.385 & 1.399 \\
Cervical & 1.368 & 1.392 \\
\hline
\end{tabular}

finement at the boundary. We analyze the frequency shift in the transmission spectra of the biosensor by varying the sample RI. In Fig. 8(a), the transmission spectra have a blue-shift by increasing the sample RI from 1.33 to 1.39 , for $E_{\mathrm{f}}=0.61 \mathrm{eV}$ and the sensitivity in Fig. 8(b) is $407.7 \mathrm{THz} / \mathrm{RIU}$. This is a high sensitivity compared to the previous results in the literature [37].

\subsection{Comparison of the Two Configurations}

In Subsecs. 4.1 and 4.2 we presented the results of the first and second configurations separately. Both configurations show a high sensitivity compared to existing results in the literature. In this subsection, we compared the sensitivity of the two configurations.

Table 1 is the comparison between the sensitivity of the two configurations in this paper. Table 1 shows that the second configuration has a high sensitivity for the metal and graphene structures compared to the first configuration and has an acceptable sensitivity for the metamaterial structure.

Based on the summarized results in Table 1, we present the results for cancer cells in the second configuration. These cancer cells can also be tested by the first configuration, however, to avoid repeating results, we only focus on the results of the second configuration.

\subsection{Cancer Samples in the Second Configuration}

We employ three types of cancer cells, including basal, breast and cervical, as samples in the second configuration of the graphene-based biosensor structure. The RIs of the cancerous and normal basal, breast and cervical cells are listed in Table 2.

Here we examine the transmission spectra of biosensor for normal and cancerous cells at different frequencies. We also calculate $S$ for these three types of cancer cells. Figures 9(a)-(c) are the transmission spectra of breast, 
basal and cervical normal and cancerous cells for frequency range from $0.01 \omega_{\mathrm{e}}$ to $0.06 \omega_{\mathrm{e}}$. In Figs. 9(a)-(c), there is a red-shift in the transmission spectra of cancerous cells compared to the normal cells.

In Figs. 9(a)-(c) there are remarkable differences in the transmission spectra of cancerous and normal cells in the frequency range from $0.01 \omega_{\mathrm{e}}$ to $0.06 \omega_{\mathrm{e}}$. The transmission spectra of cancerous cells in Figs. 9(a),(b) is lower than that of normal cells. However in Fig. 9(c), cervical cancer cell exhibits higher transmission compared to the normal cell. Differences in the transmission spectra of cancer and normal cells have the potential to provide a method for early cancer detection based optical properties of the normal and cancer cells [38].

Figure $9(\mathrm{~d})$ is the sensitivity of biosensor for the three different cancerous cells. The amount of $S$ for $\Delta n$ of each cancer cell is calculated as follows: $\mathrm{S}=357 \mathrm{THz} / \mathrm{RIU}$ for $\Delta n=0.014$ of breast cancer cell, $\mathrm{S}=1034 \mathrm{THz} / \mathrm{RIU}$ for $\Delta n=0.02$ of basal cancer cell and $\mathrm{S}=195 \mathrm{THz} / \mathrm{RIU}$ for $\Delta n=0.024$ of cervical cancer cell.
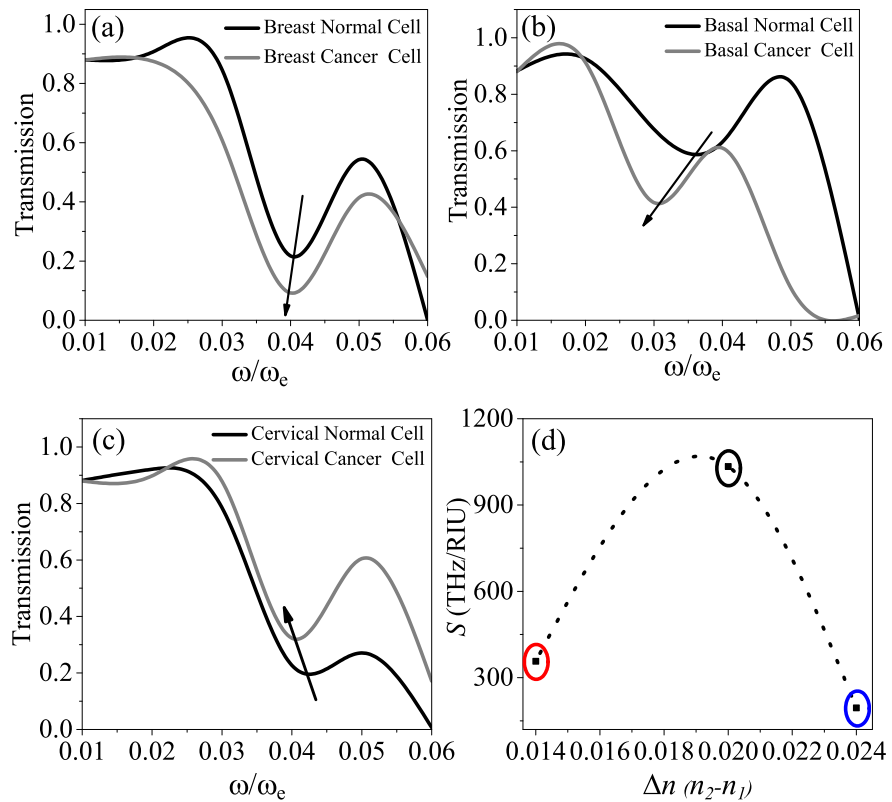

Fig. 9 The shift in the transmission spectra of biosensor for (a) breast, (b) basal and (c) cervical cancer cells (gray) versus normal cells (black), according to Table 2. Part (d) is the sensitivity of the graphene-based biosensor, when the sample is infiltrated into the sample housing for breast, basal and cervical cancer cells. The points circled with red, black, and blue represent the sensitivity for breast, basal and cervical cell, respectively.

These results verify that our plasmonic biosensor has a high sensitivity for the three types of cancerous cells compared to the previous results in the literature $[28,39]$. Table 3 is a comparison between three types of cancerous 
Table $3 S$ for three types of breast, basal and cervical cancer in second configuration of biosensor

\begin{tabular}{lll}
\hline Cell Name & $\begin{array}{l}\Delta n \\
(\mathrm{RIU})\end{array}$ & $\begin{array}{l}S \\
(\mathrm{THz} / \mathrm{RIU})\end{array}$ \\
\hline Breast & 0.014 & 357 \\
Basal & 0.02 & 1034 \\
Cervical & 0.024 & 195 \\
\hline
\end{tabular}

cells in the second configuration of the biosensor when the graphene is in the core-cladding boundary of metal-dielectric waveguides. The simulation results show a high value of $S$ for basal cancer compared to the previous research [40] that verifies our biosensor structure is more sensitive and specific for basal cancer.

\subsection{Tunability and Specificity of the Biosensor}

Another application of employing graphene in the core-cladding boundary of the metal-dielectric biosensor is to attain a tunable biosensor structure. Graphene provides a high confinement for SPP at the interface compare to metal. Moreover, graphene has a tunable surface conductivity. The surface conductivity of graphene is sensitive to $\omega, \mathrm{T}, E_{\mathrm{f}}$ and $\tau$ according to Equations (1) and (2). By applying a gate voltage to graphene, the chemical potential $E_{\mathrm{f}}$ varies. This eminent feature of graphene to tune its optical properties by applying a gate voltage motivates us to employ graphene to design a tunable biosensor.

The EM properties of graphene are modified by applying the gate voltage. The changes in EM properties of graphene affect the transmission spectra that shifts the peak or dip of the transmission spectra to different frequencies. Therefore, by applying the gate voltage, the high-sensitivity region of the biosensor shifts. Figure 10(a) is the transmission spectra of graphene-based biosensor structure with sample RI of 1.33 by varying the gate voltage. Figure 10(a) shows that the biosensor transmission spectra shifts to lower frequencies by applying different values of gate voltages. This shift in the transmission spectra shows the tunability of the biosensor sensitivity. The slope of plots in Fig. 10(b) for different $E_{\mathrm{f}}$ gives the sensitivity of the biosensor. For different RIs of samples, by applying $V_{\mathrm{g}}=2,3$ and $6 \mathrm{~V}$, the sensitivity of the biosensor decreases. 

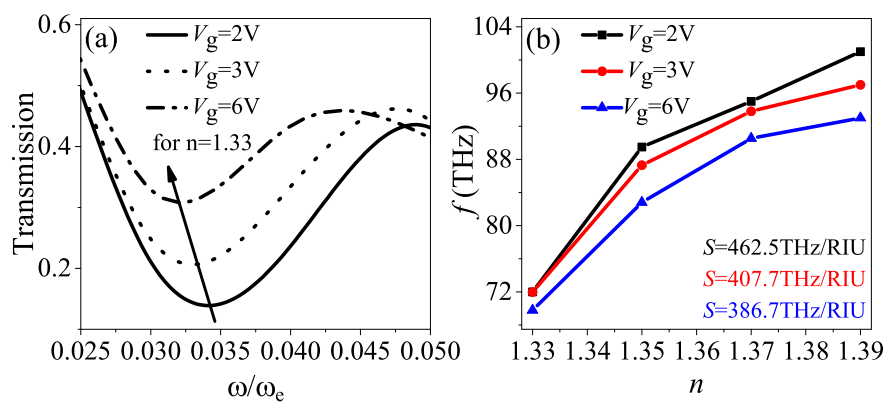

Fig. 10 (a) Transmission spectra of biosensor with the sample RI of 1.33 and (b) sensitivity of the graphene-based biosensor in the second configuration, when the sample RI changes from 1.33 to 1.39 for gate voltage $V_{\mathrm{g}}=2,3$ and $6 \mathrm{~V}$. The black, red and blue graphs in (b) correspond to the frequency shifts for $V_{\mathrm{g}}=2,3$ and $6 \mathrm{~V}$, respectively.

The analytical specificity of a biosensor is its ability to measure one particular sample among samples with RIs near to each others [41]. To distinguish the normal and cancer cells, we require biosensors with high specificity. The reason we need the biosensors with high specificity is that some normal healthy cells in the body have high growth rates like the cancerous cells. Therefore, their RI is near to the RI of cancerous cells. Here, we discuss the specificity of our biosensor structure.

To check the specificity of our biosensor, we employ normal healthy cells with RIs near to the cancer cells in Table 2 and with high growth rates such as intestinal epithelium cell, oral squamous cell, and hair follicle stem cells. The RI for the oral squamous cell is $\simeq 1.4$ [42], for intestinal epithelium cell is $\simeq 1.035$ [43] and for hair follicle stem cells is $\simeq 1.37$ [44]. Although cancer cells including the cells listed in the Table 2, have RIs near to the RIs of these high growth rates cells, our structure has the capability to distinguish between these two normal and cancer cells because of shift in the transmission spectra of the sensor. Therefore, we can conclude that our biosensor has an acceptable specificity.

\section{Conclusion}

In this paper, we design and simulate a biosensor structure made of plasmonic waveguides based on asymmetric MZI. The biosensor structure is composed of linear, homogeneous, isotropic lossy dispersive media interfaces with lossless non-dispersive media. As examples of media, we employ metamaterial, metal and graphene interfaces with air and silicon.

We suggest two configurations for our biosensor structure, the first configuration is an open-path structure and the second configuration includes a sample housing. The first biosensor configuration has application for liquid, gas and solid samples, while the second configuration is used for liquid and gas samples. We employ samples with RIs ranging from 1.33 to 1.39, as 
instances. A biosensor operation at this RI range has applications in environmental, chemical and biochemical sensing. However, our structure is not limited to this specific range of the sample RI and can accommodate media with different RIs.

We embed the structure in the sample and examine the sensing performance of the biosensor at different conditions. By changing the sample RI, the biosensor transmission spectra shifts to different frequencies. From the changes in the transmission spectra, we detect cancerous cells. We employ three types of cancer cells as samples for the biosensor, including basal, breast and cervical cancer cells. This biosensor structure is designed for early detecting of cancer cells to have rapid treatments. The sensitivities of our biosensor for breast, basal and cervical cancer types are 357, 1034 and 195THz/RIU, respectively. These amount of sensitivity are higher than previously reported results.

Our biosensor is specific that is concluded from a comparison between basal, breast and cervical cancer cells with healthy intestinal epithelium cell, oral squamous cell and hair follicle stem cells. To attain a tunable biosensor, we employ graphene and take the advantage of graphene sensitivity to the gate voltage. By applying a gate voltage to the graphene in the biosensor structure, the transmission spectra of the biosensor shifts to different frequencies that affect the sensitivity of the biosensor.

\section{Funding}

There is no Fund.

\section{Conflict of interest/Competing interests}

The authors declare that they have no conflict of interest.

\section{Avalibility of data and material}

Not applicable.

\section{Code availibility}

Not applicable.

\section{Author's contributions}

Conception and design of study is made by Fariba Lotfi, Nafiseh Sang-Nourpour, Reza Kheradmand. Fariba Lotfi designed the simulation and wrote the draft- 
ing of manuscript. Fariba Lotfi, Nafiseh Sang-Nourpour Analyzed the simulation data. Fariba Lotfi, Nafiseh Sang-Nourpour, Reza Kheradmand revised the manuscript critically for important intellectual content.

\section{Ethics approval}

Not applicable.

\section{Consent to participate}

Not applicable.

\section{Consent for publication}

Not applicable.

\section{References}

1. Momota, Moriom Rojy and Hasan, Md Rabiul, Hollow-core silver coated photonic crystal fiber plasmonic sensor, Optical Materials, 76, 287-294 (2018)

2. Myszka, David G and Rich, Rebecca L, Implementing surface plasmon resonance biosensors in drug discovery, Pharmaceutical science \& technology today, 3, 310-317 9 (2000)

3. Situ, Chen and Mooney, Mark H and Elliott, Christopher T and Buijs, Jos, Advances in surface plasmon resonance biosensor technology towards high-throughput, food-safety analysis, TrAC Trends in Analytical Chemistry, 29, 1305-1315 11 (2010)

4. Ligler, Frances S and Taitt, Chris Rowe and Shriver-Lake, Lisa C and Sapsford, Kim E and Shubin, Yura and Golden, Joel P, Array biosensor for detection of toxins, Analytical and bioanalytical chemistry, 377, 469-477 3 (2003)

5. Wu, Leiming and Guo, Jun and $\mathrm{Xu}$, Hailin and Dai, Xiaoyu and Xiang, Yuanjiang, Ultrasensitive biosensors based on long-range surface plasmon polariton and dielectric waveguide modes, Photonics Research, 4, 262-266 6 (2016)

6. Zhang, Zhaojian and Yang, Junbo and He, Xin and Zhang, Jingjing and Huang, Jie and Chen, Dingbo and Han, Yunxin, Plasmonic refractive index sensor with high figure of merit based on concentric-rings resonator, Sensors, 18, 1161 (2018)

7. Sang-Nourpour, Nafiseh and Lavoie, Benjamin R and Kheradmand, Reza and Rezaei, M and Sanders, Barry C, Characterization of surface-plasmon polaritons at lossy interfaces, Journal of Optics, 19, 12500412 (2017)

8. Khan, Asad and Krupin, Oleksiy and Lisicka-Skrzek, Ewa and Berini, Pierre, MachZehnder refractometric sensor using long-range surface plasmon waveguides, Applied Physics Letters, 103, 11110811 (2013)

9. Vernon, Kristy C and Gómez, Daniel E and Davis, Timothy J, A compact interferometric sensor design using three waveguide coupling, Journal of Applied Physics, 106, 10430610 (2009)

10. Hong, Jongin and Choi, Jung Sung and Han, Gayoung and Kang, Jae Kwang and Kim, Chang-Min and Kim, Tae Song and Yoon, Dae Sung, A Mach-Zehnder interferometer based on silicon oxides for biosensor applications, Analytica chimica acta, 573, 97-103 (2006) 
11. Liu, Qing and Tu, Xiaoguang and Kim, Kyung Woo and Kee, Jack Sheng and Shin, Yong and Han, Kyungsup and Yoon, Yong-Jin and Lo, Guo-Qiang and Park, Mi Kyoung, Highly sensitive Mach-Zehnder interferometer biosensor based on silicon nitride slot waveguide, Sensors and Actuators B: Chemical, 188, 681-688 (2013)

12. Chen, Yangyang and Liu, Jinchuan and Yang, Zhenchuan and Wilkinson, James S and Zhou, Xiaohong, Optical biosensors based on refractometric sensing schemes: A review, Biosensors and Bioelectronics, 144, 111693 (2019)

13. Bohunicky, Brian and Mousa, Shaker A, Biosensors: the new wave in cancer diagnosis, Nanotechnology, science and applications, 4, 1 (2011)

14. Ishimaru, Akira, Wave propagation and scattering in random media, 336-393. Academic press New York, New York (1978)

15. Beig-Mohammadi, Maryam and Sang-Nourpour, Nafiseh and Sanders, Barry C and Lavoie, Benjamin R and Kheradmand, Reza, Hybrid mode tunability in metamaterial nanowaveguides, Optical Engineering, 56, 0271052 (2017)

16. Lotfi, Fariba and Sang-Nourpour, Nafiseh and Kheradmand, Reza, Plasmonic all-optical switching based on metamaterial/metal waveguides with local nonlinearity, Nanotechnology, 31, 0152011 (2019)

17. Sang-Nourpour, Nafiseh and Lavoie, Benjamin R and Kheradmand, R and Rezaei, M and Sanders, Barry C, Electromagnetic-magnetoelectric duality for waveguides, arXiv preprint arXiv:1510.06458, (2015)

18. Palik, Edward D, Handbook of optical constants of solids, 28. Academic press, Boston (1998)

19. Shen, Le and Shen, Hui-Shen and Zhang, Chen-Li, Temperature-dependent elastic properties of single layer graphene sheets, Materials \& design, 31, 4445-4449 9 (2010)

20. Rodrigo, Daniel and Limaj, Odeta and Janner, Davide and Etezadi, Dordaneh and De Abajo, F Javier García and Pruneri, Valerio and Altug, Hatice, Mid-infrared plasmonic biosensing with graphene, Science, 349, 165-168 6244 (2015)

21. Grigorenko, Alexander N and Polini, Marco and Novoselov, KS, Graphene plasmonics, Nature photonics, 6, 749-758 11 (2012)

22. $\mathrm{Xu}$, Chao and Jin, Yichang and Yang, Longzhi and Yang, Jianyi and Jiang, Xiaoqing, Characteristics of electro-refractive modulating based on Graphene-Oxide-Silicon waveguide, Optics express, 20, 22398-22405 20 (2012)

23. Chen, Fang and Yao, Duanzheng and Liu, Yanan, Graphene-metal hybrid plasmonic switch, Applied Physics Express, 7, 0822028 (2014)

24. Špačková, Barbora and Wrobel, Piotr and Bocková, Markéta and Homola, Jiří, Optical biosensors based on plasmonic nanostructures: a review, Proceedings of the IEEE, 104, 2380-2408 12 (2016)

25. Cai, Wenshan and Shalaev, Vladimir M, Optical metamaterials, 101-122. Springer, New York (2010)

26. Salihoglu, Omer and Balci, Sinan and Kocabas, Coskun, Plasmon-polaritons on graphene-metal surface and their use in biosensors, Applied Physics Letters, 100, 213110 $21(2012)$

27. Lotfi, Fariba and Sang-Nourpour, Nafiseh and Kheradmand, Reza, High-sensitive plasmonic sensor based on Mach-Zehnder interferometer, Optics \& Laser Technology, 137, 106809 (2021)

28. Ayyanar, N and Raja, G Thavasi and Sharma, Mohit and Kumar, D Sriram, Photonic crystal fiber-based refractive index sensor for early detection of cancer, IEEE sensors journal, 18, 7093-7099 17 (2018)

29. Omri, Mohamed and Ouerghi, Faouzi and Abdelmalek, Fathi and Haxha, Shyqyri, Highly Sensitive Photonic Sensor Based on V-Shaped Channel Mediated Gold Nanowire, IEEE sensors journal, 20, 8505-8511 15 (2020)

30. Saadeldin, A Samy and Hameed, Mohamed Farhat O and Elkaramany, Essam MA and Obayya, Salah SA, Highly sensitive terahertz metamaterial sensor, IEEE sensors journal , 19, 7993-7999 18 (2019)

31. Nejat, Mohamad and Nozhat, Najmeh, Ultrasensitive THz refractive index sensor based on a controllable perfect MTM absorber, IEEE sensors journal, 19, 10490-10497 22 (2019)

32. Cen, Chunlian and Lin, Hang and Huang, Jing and Liang, Cuiping and Chen, Xifang and Tang, Yongjian and Yi, Zao and Ye, Xin and Liu, Jiangwei and Yi, Yougen and others, 
A tunable plasmonic refractive index sensor with nanoring-strip graphene arrays, Sensors, $18,448922(2018)$

33. An, Guowen and Li, Shuguang and Wang, Haiyang and Zhang, Xuenan, Metal oxidegraphene-based quasi-D-shaped optical fiber plasmonic biosensor, IEEE Photonics Journal, 9, 1-9 4 (2017)

34. Kieu, K and Schneebeli, L and Norwood, RA and Peyghambarian, N, Integrated liquidcore optical fibers for ultra-efficient nonlinear liquid photonics, Optics express, 20, 8148 81547 (2012)

35. Wang, Ying and Liao, CR and Wang, DN, Femtosecond laser-assisted selective infiltration of microstructured optical fibers, Optics express, 18, 18056-18060 17 (2010)

36. Chou Chau, Yuan-Fong and Chou Chao, Chung-Ting and Huang, Hung Ji and Kumara, NTRN and Lim, Chee Ming and Chiang, Hai-Pang, Ultra-high refractive index sensing structure based on a metal-insulator-metal waveguide-coupled T-shape cavity with metal nanorod defects, Nanomaterials, 9, 143310 (2019)

37. Akbari, Ladan and Abedi, Kambiz, A highly sensitive and tunable plasmonic sensor based on a graphene tubular resonator, Optics Communications, 458, 124686 (2020)

38. Salomatina, Elena Vladimirovna and Jiang, Brian and Novak, John and Yaroslavsky, Anna N, Optical properties of normal and cancerous human skin in the visible and nearinfrared spectral range, Journal of biomedical optics, 11, 0640266 (2006)

39. Hlali, Aymen and Oueslati, Afef and Zairi, Hassen, Numerical Simulation of Tunable Terahertz Graphene-Based Sensor for Breast Tumor Detection, IEEE Sensors Journal, 21 9844-9851 8 (2021)

40. Azab, Mohammad Y and Hameed, Mohamed Farhat O and Nasr, Abed M and Obayya, SSA, Highly Sensitive Metamaterial Biosensor for Cancer Early Detection, IEEE Sensors Journal, 21, 7748-7755 6 (2021)

41. Saah, Alfred J and Hoover, Donald R, "Sensitivity" and "specificity" reconsidered: the meaning of these terms in analytical and diagnostic settings, Annals of internal medicine, 91-94 1 (1997)

42. Einstein, Gnanatheepam and Udayakumar, Kanniyappan and Aruna, Prakasa Rao and Koteeswaran, Dornadula and Ganesan, Singaravelu, Diffuse reflectance spectroscopy for monitoring physiological and morphological changes in oral cancer, Optik, 127, 1479-1485 (2016)

43. Backman, Vadim and Gurjar, Rajan and Badizadegan, Kamran and Itzkan, Irving and Dasari, Ramachandra R and Perelman, Lev T and Feld, Michael S, Polarized light scattering spectroscopy for quantitative measurement of epithelial cellular structures in situ, IEEE Journal of Selected Topics in Quantum Electronics, 5, 1019-1026 4 (1999)

44. Huang, Xiyong and Protheroe, Michael D and Al-Jumaily, Ahmed M and Paul, Sharad $\mathrm{P}$ and Chalmers, Andrew N, Review of human hair optical properties in possible relation to melanoma development, Journal of biomedical optics, 23, 0509015 (2018) 